\section{LA-UR- 02, -6878}

Approved for public release; distribution is unlimited.

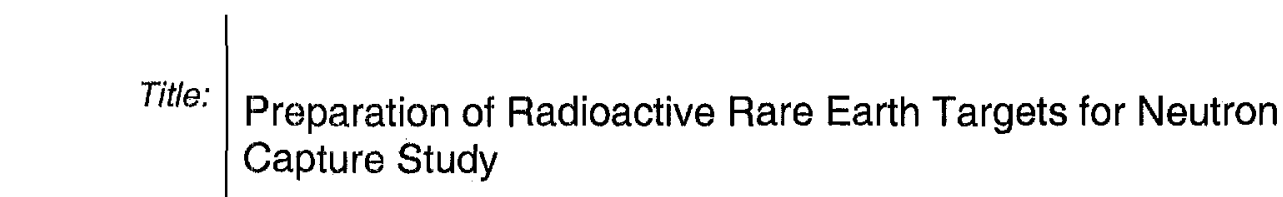

Author(s):

G.G. Miller, P.Z. Rogers, P.D. Palmer, D.E. Dry, R.S. Rundberg, M.M. Fowler, J.B. Wilhelmy

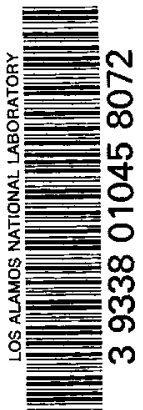

Submitted to:

Presented at the MARC VI conference on "Methods and Applications of Radioanalytical Chemistry" 


\title{
Preparation of Radioactive Rare Earth Targets for Neutron Capture Study
}

\author{
G.G. Miller, P.D. Palmer, D.E. Dry, R. S. Rundberg, M.M. Fowler, J.B. Wilhelmy \\ Los Alamos National Jaboratory, Los Alamos, NM 87545
}

\section{Introduction}

The understanding of the details of nucleosynthesis in stars remains a great challenge. Though the basic mechanisms governing the processes have been known since the pioneering work of Burbidge, Burbidge, Fowler and Hoyle (1), we are now evolving into a condition where we can ask more specific questions. Of particular interest are the dynamics of the s ("slow") process. In this process the general condition is one in which sequential neutron captures occur at time scales long compared with the beta decay half lives of the capturing nuclides. The nucleosynthesis period for $\mathrm{C}$ or Ne burning stellar shells is believed to be in the year to few year time frame (2). This means that radionuclides with similar half lives to this burning period serve as "branch point" nuclides. That is, there will be a competition between a capture to the next heavier isotope and a beta decay to the element of next higher atomic number. By understanding the abundances of these competing reactions we can learn about the dynamics of the nucleosynthesis process in the stellar medium. Crucial to this understanding is that we have a knowledge of the underlying neutron reaction cross sections on these unstable nuclides in the relevant stellar energy regions (neutrons of $0.1-100 \mathrm{KeV}$ ). ${ }^{171} \mathrm{Tm}$ ( 1.9 years) and ${ }^{151} \mathrm{Sm}$ (90 years) have decay properties that permit their handling in an open fume hood. These two were therefore selected to be the first radionuclides for neutron capture study in what will be an ongoing effort.

The pulsed, time-of-flight neutron source at the Los Alamos Neutron Science Center (LANSCE) is described elsewhere (3). The basic experiment for which these REE sources was prepared was for realtime recording of capture events through the detection of prompt gamma rays. In order to make suitable targets for this type of experiment, two main issues had to be addressed:

a.) Chemical purification of the REE's

b. ) Mounting the REE's, at mass densities of $\approx 1 \mathrm{mg} / \mathrm{cm}^{2}$, in such a way that background captures would be kept to a minimum.

Most of the work required to prepare an acceptable target of a radioactive rare earth isotope lies in its separation from other REE's; must notably from its daughter and any target material from which it was prepared. Fast reliable separations of REE's, on a laboratory scale, are most often accomplished by cation exchange chromatography in the presence of methyl lactic acid (MLA), aka $\alpha$-hydroxyisobutyric acid. Such work is carried out by either "classical" ion exchange (4-8) or by the more efficient "dynamic" ion exchange technique pioneered in the 1980's (9-12). In the former, a column with fixed exchange sites is employed. In the latter, a modifier (sometimes referred to as an "ion-pairing" agent) such as sodium octanesulfonate, is added to the mobile phase in order to generate in-situ ion exchange sites in a reversedphase column. While both methods have worked well for us in analytical studies, we opted to use the "classical" method for this work. Probably because of the low (linear) solvent flow rates that we employed, the poor column resolution experienced by others wasn't observed. $(9,10)$

Once sufficiently pure rnaterial is available for study, targets for real-time neutron capture measurements must be carefully prepared. Any extraneous mass, for example backing or support material, will introduce background, either directly by neutron capture, or indirectly through the scattering of neutrons that subsequently capture in surrounding materials (including the detectors themselves). Since the neutron fluxes were low (approx. $10^{6}$ neutrons $/ \mathrm{cm}^{2} / \mathrm{sec}$ ) in the energy ranges of interest, it was necessary to have targets containing milligram (i.e. $10^{18}$ atoms) amounts of material to ensure adequate capture rates. To prepare such thick targets on thin backing foils proved difficult.

\section{Experimental}

All HPLC separations were accomplished using a Waters 600E Multisolvent Delivery System (Waters Corp., Milford, MA). The unit was equipped with a model U6K injector having a sample loop that allowed injection of up to $2 \mathrm{ml}$ of solution. The REE's were detected by post-column derivitization with Arsenazo III, followed by absorbance measurement of the resulting complexes. The detector was a Waters 
model 441 fitted with a filter to isolate the $658 \mathrm{~nm} \mathrm{Hg} \mathrm{line.} \mathrm{The} \mathrm{Arsenazo} \mathrm{III} \mathrm{was} \mathrm{delivered} \mathrm{using} \mathrm{a} \mathrm{Gilson}$ model 305 single piston reciprocating pump (Gilson, Middleton, WI). The mixing " $\mathrm{T}$ " was purchased from Upchurch Scientific (Oak Harbor, WA). Two columns were employed; a $10 \mathrm{~mm}$ i.d. x $250 \mathrm{~mm} 5 \mu \mathrm{m}$ Capcell SCX column (purchased through Phenomenex Corp. Torrance, CA), and a $10 \mathrm{~mm} \times 250 \mathrm{~mm}$ column (Phenomenex) packed with AG50x8 325 mesh cation resin (Bio-Rad, Hercules, CA). A flow rate of $3 \mathrm{ml} / \mathrm{min}$ was used with both columns. The Arsenazo reagent $\left(1.5 \times 10^{-4} \mathrm{M}\right)$ was delivered at 1.5 $\mathrm{ml} / \mathrm{min}$.

Methyl lactic acid (MLA) was obtained from Acros Chemicals (Fisher Scientific, Pittsburgh, PA) and was used without further purification. Arsenazo III [2,2'-(1,8-dihydroxy-3,6-disulfonaphthalene-2,7bisazo)bis-benzenearsonic acid], was purchased from Alfa Aesar (Ward Hill, MA) and was used without further purification. All HPLC solutions were filtered through a $0.45 \mu \mathrm{m}$ filter and de-gassed by sonication under vacuum immediately prior to use.

Electrodepositions were carried out using a Pine Instruments Corp. (Grove City, PA) model AFCPRB rotating electrode and an HP 6113A (Hewlett Packard, Palo Alto, CA) regulated DC power supply. A 0.125 inch diameter $\mathbf{P}$ rod served as both stirrer and anode. The electroplating cell consisted of a 0.375 inch i.d. glass chimney that was pressed onto the Be/Ti cathode via a spring mechanism. A flexible PVC gasket rendered the chimney/cathode seal water-tight. Plating was done from approximately $4 \mathrm{ml}$ of $0.5 \mathrm{M} \mathrm{NaHSO}_{4}$ solution adjusted to $\mathrm{pH} 2.0$. Plating was carried out for 25 minutes at a current density of $0.5 \mathrm{~A} / \mathrm{cm}^{2}$ and at an anode rotation rate of $1400 \mathrm{rpm}$.

\section{Results and Discussion}

\section{Tm production/purification}

Approximately 0.5 grams of $\mathrm{Er}_{2} \mathrm{O}_{3}$, enriched to $97 \%$ in ${ }^{170} \mathrm{Er}$ (Oak Ridge National Laboratory) were sealed in high purity synthetic quartz and irradiated for $\mathbf{4 0}$ days in the research reactor at the Idaho National Engineering Laboratory. About $3 \mathrm{mg}$ of ${ }^{171} \mathrm{Tm}$ were produced. After allowing ${ }^{170} \mathrm{Tm}$ to decay for $\approx 5$ months, the $\mathrm{Er}_{2} \mathrm{O}_{3}$ was dissolved in $\mathrm{HCl}$ and converted to nitrate form by repeated fuming with concentrated $\mathrm{HNO}_{3}$. The sample was taken-up into $\approx 20 \mathrm{ml}$ of $0.3 \mathrm{M} \mathrm{HNO}_{3}$ in preparation for HPLC fractionation.

The high-resolution $10 \mathrm{~mm} \times 250 \mathrm{~mm}$ Capcell column could, at best, accommodate only 2-3 mg of rare earth per injection. Given the large mass of Er present, a column of much greater capacity was required. Thus, a $10 \mathrm{~mm} \times 250 \mathrm{~mm}$ steel column, packed with AG50x8 (325 mesh) was prepared. Because of the nearly identical chemical behavior of the heavy rare earths, separating them one from another is one of the most difficult separation problems in inorganic chemistry. This fact, coupled with the poorer resolution obtainable with the AG.50 column, required the Tm to be passed through the column three times to obtain adequate purity. Chromatograms of this sequential clean-up are shown in Figure 1, with chromatographic parameters given in the legend. Of particular importance to the separation was that it was carried out at $80^{\circ} \mathrm{C}$. According to Shuheng et al (6), running the column at elevated temperature markedly improves resolution of the heavy rare earths by decreasing both peak tailing and the peak widths at half-height. We found this to be true, but one does pay a price of significantly increased retention volumes relative to those obtained under identical conditions at room temperature. Nonetheless, the added resolution afforded by funning at high temperature was very important and run times were kept below about 1 hour by increasing the slope of the MLA gradient. It should be noted here that 10 runs like that in Figure 1a, each involving the injection of $\approx 50 \mathrm{mg}$ of Er, were required to remove the majority of the erbium target material. Because of tailing of the Tm into the Er peak, small amounts of Er were collected with each of the Tm fractions in steps 1 and 2 . This was necessary to avoid the loss of valuable Tm.

Figrure 1. Sequential clean-up of ${ }^{17 l} \mathrm{Tm}$. Gradient: 0.04 M MLA ("A") to 0.75 M MLA ("B") as shown in I.a. $\mathrm{pH}=5.7$, Temp. $=85^{\circ} \mathrm{C}$ I.a. $=1^{\text {st }}$ pass, I.b. $=2^{\text {nd }}$ pass, I.c. $=3^{\text {rd }}$ pass 
I.a.

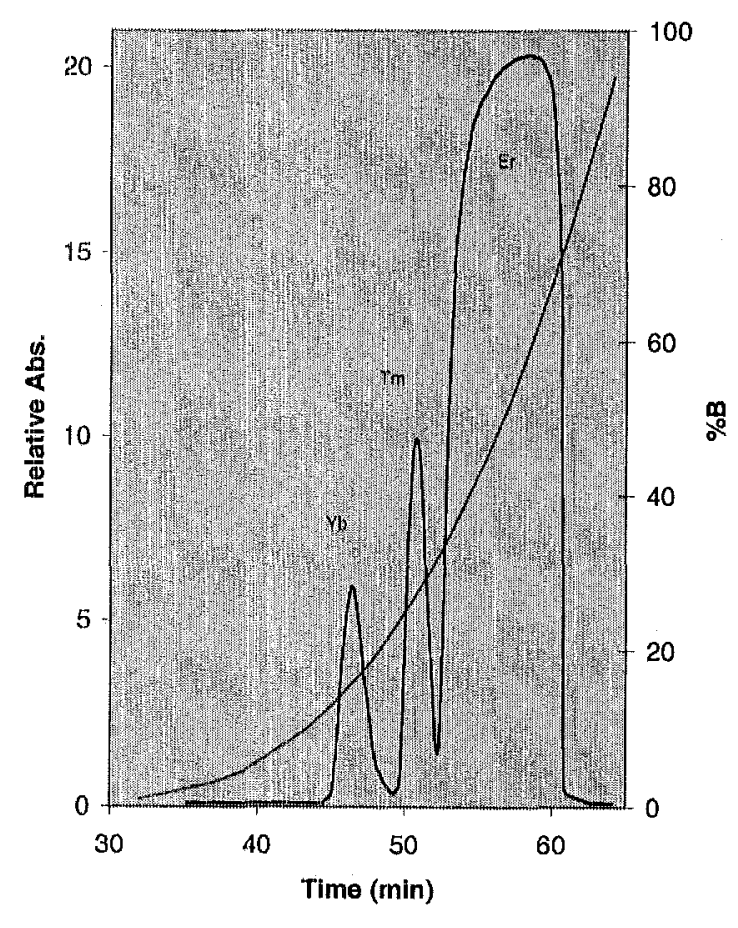

I.b.

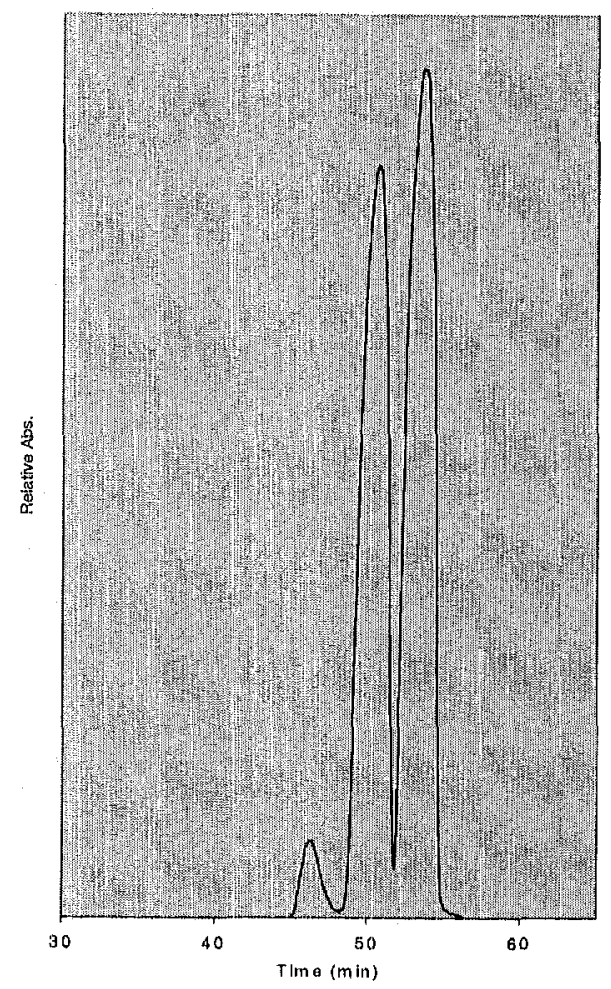


I.c.

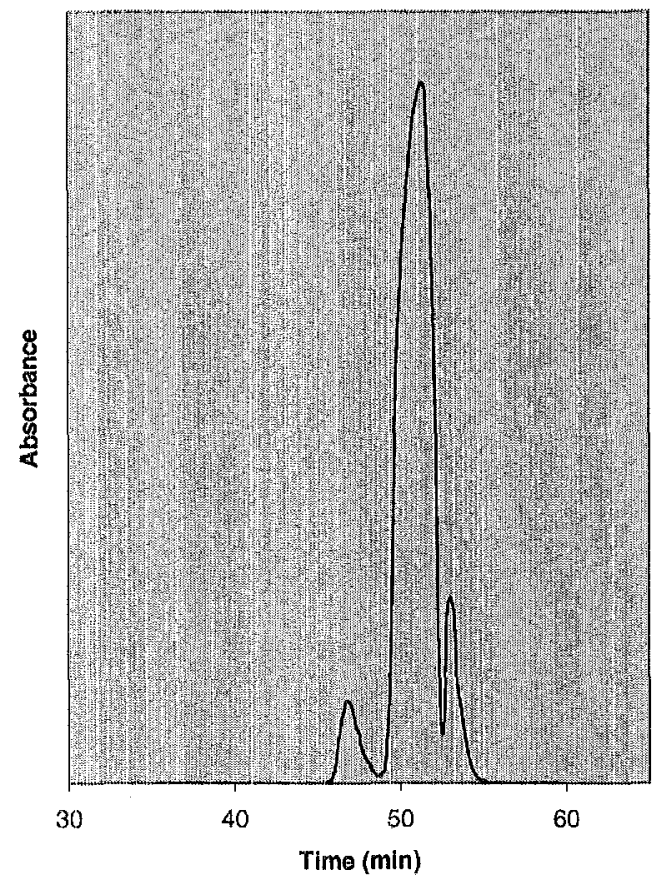

Figure $2 \mathrm{a}$ illustrates the improved resolution afforded by the Capcell column. The chromatogram in Figure $2 \mathrm{~b}$ is of an aliquot of ${ }^{171} \mathrm{Tm}$ run $\approx 18$ months after the separations of Figure 1 (hence the presence of considerable ${ }^{171} \mathrm{Yb}$ daughter). Note that the residual Er left in the ${ }^{171} \mathrm{Tm}$ target was $<0.5 \%$ by weight.

Figure 2a. Separation of selected REE's (50 $\mu \mathrm{g}$ each) using $10 \times 250 \mathrm{~mm} \times 5 \mu \mathrm{m}$ Capcell SCX column 


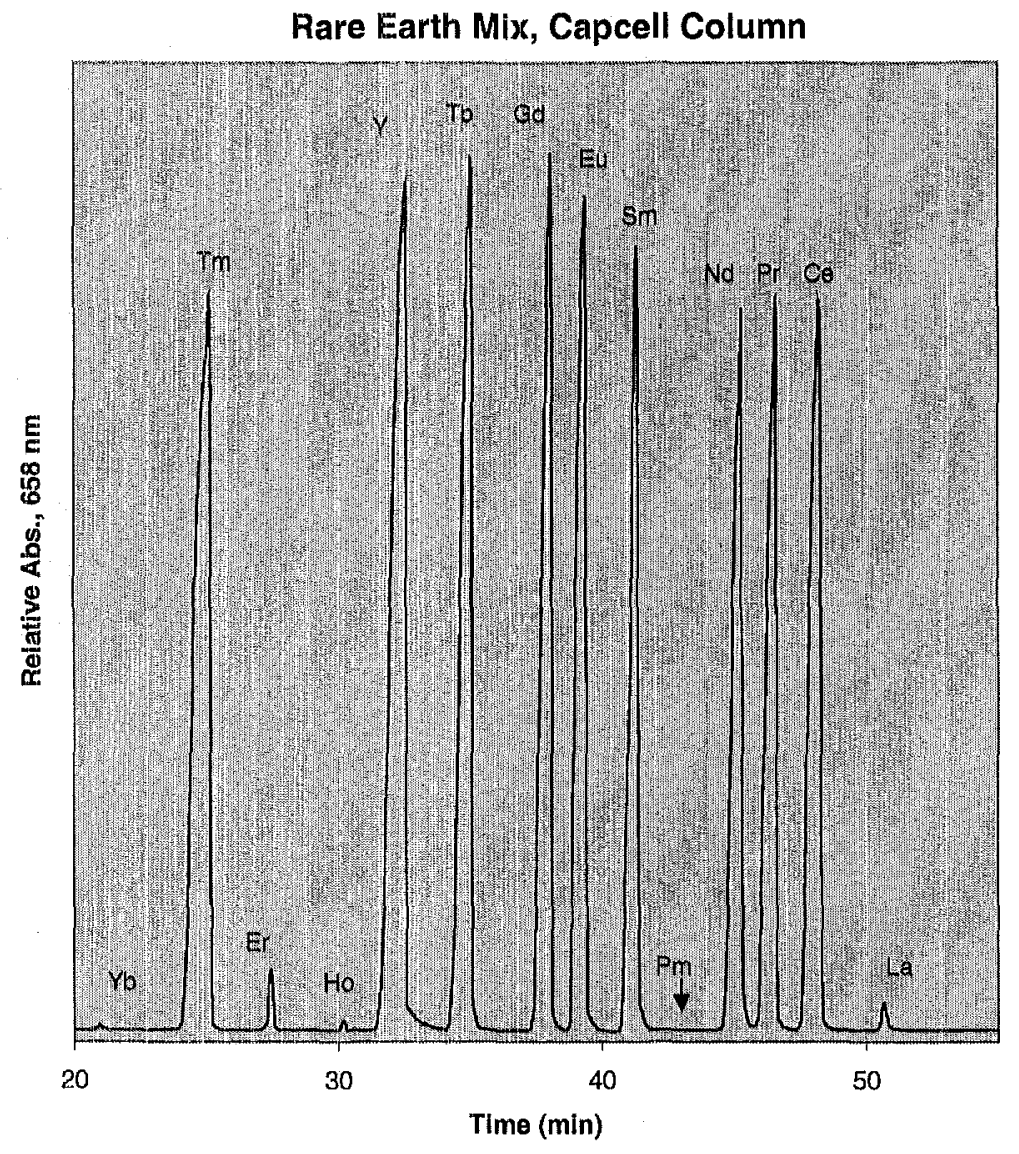

Figure $2 \mathrm{~b}$. Removal of ${ }^{171} \mathrm{Yb}$ daughter from ${ }^{171} \mathrm{Tm} 18$ months after the separations in Figure 1. Conditions: Capcell SCX column, 98\% 0.04 M MLA/2\% 0.75 M MLA to 100\% 0.75 M MLA in 25 min., pH 4.0 
Tm Purification (after 2 yr decay), Capcell column

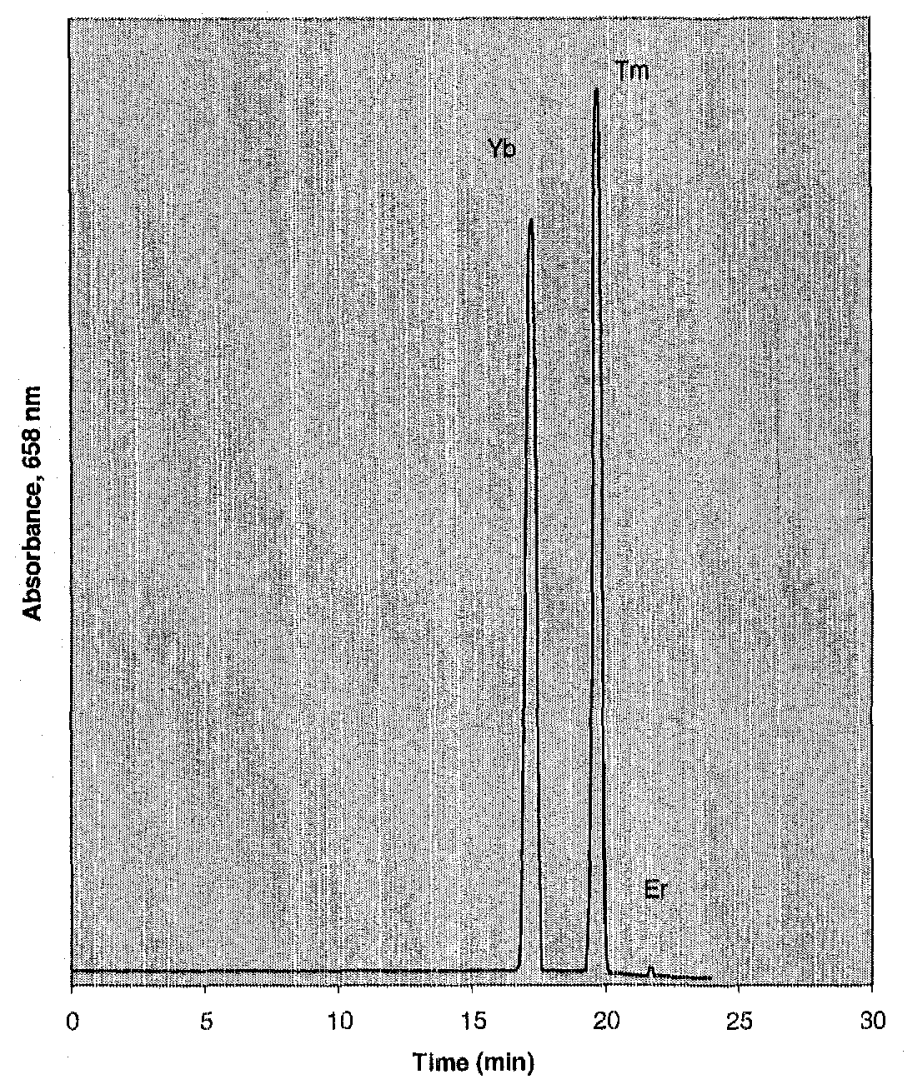

Between each of the 3 HPLC steps represented in Figure 1, the Tm had to be separated from the MLA and Arsenazo III added during processing. This was accomplished by cation exchange on a $1.5 \times 5$ $\mathrm{cm}$ column containing 50-100 mesh AGMP50. The Tm fraction was acidified to $2 \mathrm{M}$ in $\mathrm{HCl}$ from which the Tm sorbed onto the colurnn. After washing with 3-5 column volumes of $2 \mathrm{M} \mathrm{HCl}$, the Tm was eluted with 20 column volumes of $8 \mathrm{M} \mathrm{HCl}$. This was repeated twice between successive HPLC steps.

\section{Sm Purification}

Approximately $1 \mathrm{mg}$ of ${ }^{151} \mathrm{Sm}$ was obtained from Oak Ridge National Laboratory. It was dissolved in $\approx 2 \mathrm{ml}$ of $0.3 \mathrm{M} \mathrm{HNO}_{3}$ and the Eu daughter removed by HPLC. The resulting chromatogram is shown as Figure 3 with parameters given in the legend.

Figure 3. ${ }^{151} \mathrm{Sm}$ purification. Conditions: Capcell SCX column, 90\% 0.04 M MLA/10\% 0.75 M MLA to $100 \% 0.75 \mathrm{M}$ MLA in 22 min., pH 4.0 


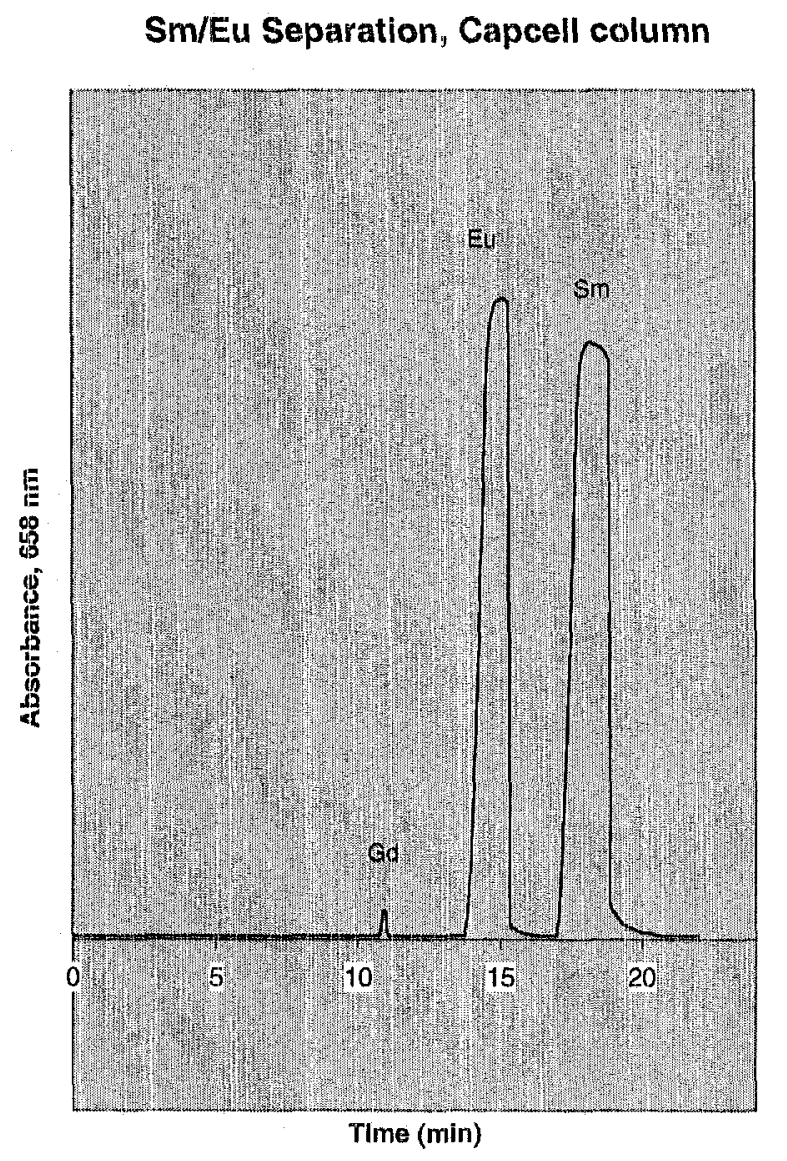

Removal of MLA and Arsenazo III from the ${ }^{151} \mathrm{Sm}$ was accomplished the same way as for the Tm. The lighter REE's however, have significantly higher $K_{d}$ 's for sorption onto cation resins from $\mathrm{HCl}$ than do their heavier congeners. Therefore considerably more $8 \mathrm{M} \mathrm{HCl}$ (approximately 40 column volumes) was required to quantitatively elute the $\mathrm{Sm}$ from the clean-up column.

\section{Source Fabrication}

For reliable capture measurements to be made, the neutron flux across the target must be uniform. The beam profile in the position to be occupied by our targets was such that an $11 \mathrm{~mm}$ diameter REE deposit was sufficient. Although preparation of free-standing metal targets would have been ideal, this was not an option given our limited quantities of material. Instead, two other options were persued; a.) mounting of a hydroxide precipitate, and b.) electrodeposition of a hydrated oxide onto Be foil. Although both choices involve the introduction of unwanted hydrogen into the neutron beam, the amounts were considered tolerable.

Stable ${ }^{169} \mathrm{Tm}$ and ${ }^{165} \mathrm{Ho}$ were used as standards. Targets containing these nuclides were made by precipitating the REE's as hydroxides with ammonia, and filtering these onto thin $0.4 \mu \mathrm{m}$ polycarbonate filters. The mass densities of both the filter $\left(0.83 \mathrm{mg} / \mathrm{cm}^{2}\right)$ and stretched mylar cover film $\left(0.10 \mathrm{mg} / \mathrm{cm}^{2}\right)$ were considered as small as practically acheivable for this type of mount. These targets behaved well, with the mounting materials having little effect upon the measured capture rates. However, this technique proved impractical when the $2 \mathrm{Ci}^{177} \mathrm{Tm}$ target was prepared. The high $\beta^{-}$radiation field caused the assembly to structurally degrade within hours of fabrication.

Attempts were then made to electrodeposit $\mathrm{Tm}$ onto Be. Trial deposits adhered very poorly and were unacceptable for use. Fortunately, other preliminary studies had yielded good REE deposits on 
titanium backing. Excellent sources were subsequently prepared by electrodeposition of REE's, including ${ }^{171} \mathrm{Tm}$, onto Be foils that had been flash-coated with 700 angstroms of Ti. Adherent rare earth deposits having mass densities below about $2 \mathrm{mg} / \mathrm{cm}^{2}$ were easily prepared and deployed as long as Ti served as the substrate for electrodeposition.

A preliminary version of the ${ }^{171}$ Tm neutron capture excitation function is given in reference 13 . Experiments with ${ }^{151}$ Sm are awaiting LANSCE beam time.

\section{References}

1. Burbidge, E.M., Burbidge, G.R., Fowler, W.A., Hoyle, F. "Synthesis of the Elements in Stars" Rev. Mod. Phys. 29, 547 (1957)

2. Wallerstein, G., et.al. "Synthesis of the Elements in Stars: Forty Years of Progress" Rev. Mod. Phys. 69, 995 (1997)

3. (description of beam at ER-1)

4. A. Mazzucotelli, A. Dadone, R. Frache, F. Baffi "Determination of Trace Amounts of Lanthanides in Rocks and Minerals by High-Performance Liquid Chromatography" J. Chromatog. 349, 137$142(1985)$

5. A.P. Leroex, R.T. Watkins "Analysis of Rare Earths in Geological Samples by Gradient Ion Chromatography: An Alternative to ICP and INAA" Chem. Geol. 88, 151-162 (1990)

6. J.M. Hwang, J.S. Shin, Y.C. Yeh, S.C. Wu "Determination of Rare Earths in Monazite Sand and Rare Earth Impurities in High-Purity Rare Earth Oxides by High-Performance Liquid Chromatography" Analyst 106, 869-873 (1981)

7. D.H. Sisson, V.A. Mode, D.O. Campbell "High Speed Separation of the Rare Earths by Ion Exchange, Part II" J. Chromatog. 66, 129-135 (1972)

8. Y. Shuheng, L, Fa, Z. Hongdi, L. Xeuliang, Z. Shulan "Applications of Pressurized Cation Exchange Chromatography for Fission Yield Determination" J. Radioanal. Nucl. Chem. Lett. 124(1) $187-195$ (1988)

9. R.M. Cassidy "Determination of Rare Earth Elements in Rocks by Liquid Chromatography" Chem. Geol. 67, 185-195 (1988)

10. C.H. Knight, R.M. Cassidy, B.M. Recoskie, L.W. Green "Dynamic Ion Exchange Chromatography for Determination of Number of Fissions in Thorium-Uranium Dioxide Fuels" Anal. Chem. 56, 474-478 (1984)

11. D.E. Stijfhoorn, H. Stray, H. Hjelmseth "Determination of Rare Earth Elements in High Purity Rare Earth Oxides by Liquid Chromatography, Thermionic Mass Spectrometry and Combined Liquid Chromatography/Thermionic Mass Spectrometry" Spectrochim. Acta 48B(4) 507-514 (1993)

12. Waters Corp. Ion Analysis Method \#M-305

13. R.S. Rundberg, J.B. Wilhelmy, M.M. Fowler, G.G. Miller, J.L. Ullmann, R.C. Haight "Neutron Capture on Off-Stable Nuclei" report LA-13547-SR, LANSCE Activity Report 1995-1998, Los Alamos National Laboratory, Los Alamos, NM 87545, pp. 86-87 